\title{
Lamellar Liquid Crystal Improves the Skin Retention of 3-O-Ethyl-Ascorbic Acid and Potassium 4-Methoxysalicylate In Vitro and In Vivo for Topical Preparation
}

\author{
Yuanru Li, ${ }^{1}$ Cuilian Dong, ${ }^{1}$ Dongmei Cun, ${ }^{1}$ Jie Liu, ${ }^{1}$ Rongwu Xiang, ${ }^{2}$ and Liang Fang ${ }^{1,3}$
}

Received 18 March 2015; accepted 9 June 2015; published online 8 September 2015

\begin{abstract}
The study aimed at increasing the skin retention of 3-O-ethyl-ascorbic acid (EA) and potassium 4-methoxysalicylate (4-MSK) via topical administration for effective skin-whitening. To achieve this goal, EA and 4-MSK were formulated into lamellar liquid crystalline (LLC) cream, and response surface methodology (RSM) was employed to optimize the formulation. Polarized light microscopy (PLM), differential scanning calorimetry (DSC), and rheological experiments were performed to confirm the presence of the LLC structure in the base of cream. In addition, a comparison analysis of the skin retention of the two drugs between the LLC cream and the common o/w (COW) cream was made through in vitro permeation and in vivo drug distribution experiments. As a result, the optimal formulation was defined as $1.2 \%$ of EA, $1.48 \%$ of $4-\mathrm{MSK}, 14.05 \%$ of Schercemol ${ }^{\mathrm{TM}}$ DISM Ester (DISM) as the oil, $4.0 \%$ of Emulium ${ }^{\circledR}$ Delta as the emulsifier, and $3.0 \%$ of stearyl alcohol as the co-emulsifier. In comparison with the COW cream, the LLC cream significantly increased the skin retention of EA and 4MSK both in vitro and in vivo. In conclusion, the LLC carrier serves as a promising choice for topical preparation by enhancing skin retention and providing desirable rheological characteristics.
\end{abstract}

KEY WORDS: 3- $O$-ethyl-ascorbic acid; lamellar liquid crystal; potassium 4-methoxysalicylate; response surface methodology; rheology.

\section{INTRODUCTION}

Skin pigmentation is a highly troublesome symptom, especially for women. Although numerous skin-whitening products are commercially available, they unfortunately tend to have no obvious effect or cause various safety issues, such as allergic dermatitis, skin damage, and even serious systemic toxic effect $(1,2)$.

The color of the human skin depends on the content of melanin produced by the melanocyte, which exists in the epidermal basal layer of the skin. Skin pigmentation results from abnormal accumulation of melanin. Skin-whitening action mechanisms can be divided into four categories: (1) inhibit the synthesis of tyrosinase, which is the key enzyme in melanogenesis; (2) inhibit the synthesis and metastasis of melanin; (3) turn oxidized melanin into the reduced form; and (4) accelerate the metabolism of melanin. To strengthen the effect of skin-whitening products, compound preparation containing at least two different drugs which act on two or more steps of the above pigmentation process is highly desired.

\footnotetext{
${ }^{1}$ Department of Pharmaceutical Science, School of Pharmacy, Shenyang Pharmaceutical University, 103 Wenhua Road, Shenyang, 110016, China

${ }^{2}$ School of Medical Instrument, Shenyang Pharmaceutical University, Shenyang, 110016, China.

${ }^{3}$ To whom correspondence should be addressed. (e-mail: fangliangspu@aliyun.com)
}

As skin-whitening active ingredients, both 3-O-ethyl-ascorbic acid (EA) and potassium 4-methoxysalicylate (4-MSK) can inhibit the activity of tyrosinase, thus inhibiting the synthesis of melanin. In addition, EA can also block the polymerization of dihydroxyindole, which is an intermediate in melanogenesis (3), while 4MSK mainly accelerates the metabolism of melanin by removing the outermost layer of the epidermis and normalizing the differentiation of keratinocyte (4). Based on their different action mechanisms, it is reasonable to combine them into a formulation to strengthen skin-whitening effect. To our best knowledge, there is no study regarding this combination has been done so far.

Even though EA and 4-MSK have positive skinwhitening effects, their high water solubility unfortunately indicates that they are likely difficult to permeate across the stratum corneum (SC) and deliver into the epidermis (5). A few studies (6-9) have previously reported that the LLC carrier displayed the SC penetration-enhancing ability. The LLC carrier is composed of parallel planar bilayers of amphiphilic surfactant molecules; therefore, it is supposed to possess good compatibility for hydrophilic drugs owing to the polarity part of the amphiphilic molecules. Figure 1 illustrated a schematic diagram of the LLC system with the two kinds of drugs incorporated between the bilayer. Moreover, as a kind of lyotropic liquid crystalline phase, the LLC system possesses spontaneous structural organization; thus, its production is simple and energy-saving (10). 


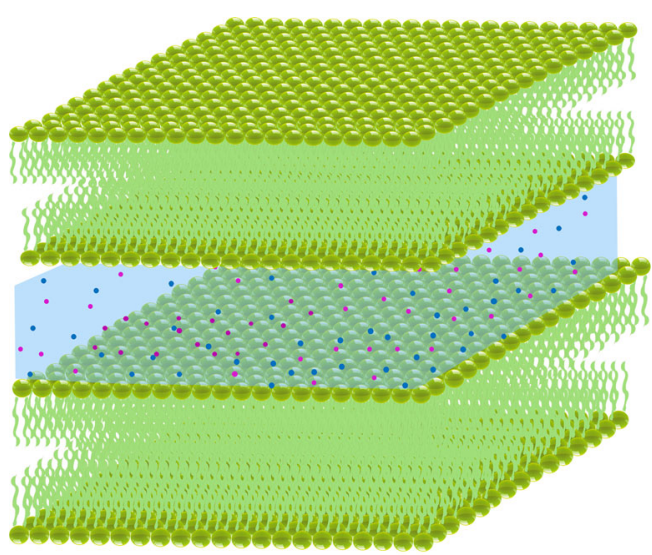

Lamellar liquid crystalline carrier

3-O-ethyl-ascorbic acid

potassium 4-methoxysalicylate

Fig. 1. A schematic diagram of the LLC system loading with EA and 4-MSK

In this study, a kind of topical LLC cream containing EA and 4-MSK was designed, and response surface methodology (RSM) was adopted to optimize the formulation in order to obtain maximum skin retention and minimum amount of penetrated drugs through the skin. Polarized light microscopy (PLM), differential scanning calorimetry (DSC), and rheological studies were carried out to prove the existence of the LLC carrier. The in vitro skin permeability and in vivo drug distribution experiments were conducted to study whether the LLC carrier can improve the skin retention or not, with the common o/w $(\mathrm{COW})$ cream as a reference.

\section{MATERIALS AND METHODS}

\section{Materials}

Emulium ${ }^{\circledR}$ Delta (INCI: cetyl alcohol, glyceryl stearate, PEG-75 stearate, Ceteth-20, and Steareth-20), Poloxamer 188, dimethicone, and stearyl alcohol were gifts from Puen Biochemical Technology Co. Ltd. (Shanghai, China). EA and 4MSK were, respectively, obtained from Dongde Chemical Technology Co. Ltd. (Nanjing, China) and Delai Biotech Co. Ltd. (Chengdu, China). Floramac Macadamia Oil (Floramac) and Florasun 90 (Florasun) were from FloraTech Co. Ltd. (Shanghai, China). Schercemol ${ }^{\mathrm{TM}}$ DIA Ester (DIA) and Schercemol ${ }^{\mathrm{TM}}$ DISM Ester (DISM) were supplied by Lubrizol Corporation (Shanghai, China). SEPIGEL305 was provided by SEPPIC Company (France). All other chemicals were of the highest reagent grade available.

\section{Animals}

Rabbits (male, 2.0-2.2 kg) and Kun Ming (KM) mice (male, 18-22 g) were supplied by the Animal Experimental Center of Shenyang Pharmaceutical University (Shenyang, China). All animal experiments were performed in strict accordance with the guidelines approved by the Life Science Research Center of Shenyang Pharmaceutical University. All efforts have been done to minimize animal suffering and to limit the number of animals sacrificed.

\section{Preparation of the LLC Cream and the COW Cream}

For the preparation of LLC cream, the oil phase consisted of various types of oil, $4.0 \%$ of the Emulium ${ }^{\circledR}$ Delta as the emulsifier and $3.0 \%$ of stearyl alcohol as the thickening agent, and the aqueous phase contained water, EA, and 4MSK. The oil phase was melted at $80^{\circ} \mathrm{C}$ in water bath, and the aqueous phase was heated to the same temperature. Then, the oil phase was added into the aqueous phase, and the mixture was kept at $80^{\circ} \mathrm{C}$ and homogenized under the speed of $8,000 \mathrm{rpm} \mathrm{min}{ }^{-1}$ for $5 \mathrm{~min}$ by using a homogenizer (FJ200$\mathrm{SH}$, Shanghai Specimen Model Factory, China) to form the emulsion. And then, the emulsion was continually stirred with a magnetic stirrer at $600 \mathrm{rpm} \mathrm{min}{ }^{-1}$ for 30 min to make the emulsion cool down and got a homogeneous cream.

In the case of COW cream, the oil phase was composed of a certain amount of oil, and the aqueous phase included EA, 4-MSK, and $4.0 \%$ of Poloxamer 188 as emulsifier. The COW cream was prepared by heating the aqueous phase and the oil phase separately to $80^{\circ} \mathrm{C}$, with Poloxamer 188 swelling in the water in advance. The following steps were the same as the LLC cream. After the formation of emulsion, 3\% of SEPIGEL305 as the thickening agent was added finally.

\section{In Vitro Skin Permeability Study}

The process of rabbit skin preparation was carried out according to our previous report (11). Full thickness skin (including the SC, epidermis, and dermis) was excised from the abdominal area and stored at $-70^{\circ} \mathrm{C}$ prior to further experiments (used within 1 month after preparation).

Skin permeation experiments were conducted through excised rabbit skin by using Franz diffusion cell systems (with an effective diffusion area of $1.54 \mathrm{~cm}^{2}$ ), which were stirred at a constant speed of $600 \mathrm{rpm}$ and thermostated with a water jacket at $32^{\circ} \mathrm{C}$. The skin was mounted between the donor and receptor part of Franz cell by clamping them, with the $\mathrm{SC}$ side of the skin facing the donor compartment. About $2 \mathrm{~g}$ of the cream was applied on the SC as the donor phase, while $7.0 \mathrm{~mL}$ of distilled water was filled into the receptor compartment. At predetermined time intervals of $2,4,6,8,10$ and $12 \mathrm{~h}$, the receptor solution of $5.0 \mathrm{~mL}$ was sampled for analysis, and an equal volume of fresh receptor medium was subsequently added to maintain sink condition. The samples were analyzed by high-performance liquid chromatography (HPLC) (Hitachi, Japan). All the permeation experiments were done in triplicate or quadruplicate.

At the end of the permeation experiment, skin was removed from the receptor cell. The residual cream on the surface of the skin was gently wiped off by a cotton swab. The effective diffusion area of the skin was cut off and accurately weighed by a one over ten thousand electronic analytical balance (AL-104 Mettler Toledo, Shanghai, China). Then, the skin was cut into very small pieces, put into a tube containing $1 \mathrm{~mL}$ of $\mathrm{pH} 7.4$ phosphate-buffered saline, and sonicated for $20 \mathrm{~min}$. The tube liquid was extracted and centrifuged (H 2050R, Xiang Yi Centrifuge Instrument Co., 
Ltd, Hunan, China) at 16,000 rpm for $5 \mathrm{~min}$. Supernatant was taken for HPLC analysis.

\section{Development of LLC Cream Formulation}

Screening of the Oil Type

Five types of oil (Floramac, Florasun, dimethicone, DIA, DISM) were prepared into corresponding LLC creams according to the approach described in the "Preparation of the LLC cream and the COW cream" section. The skin retention of the five creams was investigated through in vitro permeation experiment. The oil providing the most skin retention for both EA and 4-MSK was chosen as the formulation ingredient.

\section{Formulation Optimization by RSM}

In order to further optimize the formulation to achieve maximum skin retention and minimum penetration of drugs, a three-factor central composite design-response surface methodology (CCD-RSM) was used here. It was found in preliminary studies (data not shown here) that the concentration of drugs and the oil are the most important factors relevant to skin permeation, skin retention, and skin sensation, so EA proportion $\left(X_{1}\right)$, 4-MSK proportion $\left(X_{2}\right)$, and the oil proportion $\left(X_{3}\right)$ were identified as independent variables, and the range of the three factors was determined as follows: EA proportion (1-2\%), 4-MSK proportion (1-2\%), and oil proportion $(10-30 \%, w / w)$. The coded independent values were shown in Table I. Since cosmetic ingredients target on the skin, the optimal formulation was determined as the maximum value of skin retention of EA $\left(Q_{\mathrm{s} 1}\right)$ and 4-MSK $\left(Q_{\mathrm{s} 2}\right)$ and the minimum amounts of cumulative penetration of EA $\left(Q_{1}\right)$ and 4-MSK $\left(Q_{2}\right)$, with the least application amount of active ingredients. Therefore, $Q_{\mathrm{s} 1}, Q_{\mathrm{s} 2}, Q_{1}$, and $Q_{2}$ were defined as four response variables. Design Expert ${ }^{\circledR}$ software was employed to design and evaluate the optimal formulation.

\section{Characterization of the LLC Cream}

$P L M$

A BA300Pol polarized light microscope connected with a video camera (Motic China Group Co. Ltd., China) was used to observe the isotropic or anisotropic behavior of the samples. Each sample was uniformly applied onto a glass slide and covered with a cover slip and then analyzed under crossed polarized light with magnification of $\times 400$.
$D S C$

The thermodynamic behaviors of the LLC cream and the COW cream were studied by DSC-1 STAR ${ }^{\mathrm{e}}$ system (Mettler Toledo International Inc, Switzerland). Slightly less than $5 \mathrm{mg}$ of the sample was placed in a hermetically sealed aluminum crucible and heated from -40 to $80^{\circ} \mathrm{C}$ at a rate of $5^{\circ} \mathrm{C} \mathrm{min}{ }^{-1}$ under the $\mathrm{N}_{2}$ atmosphere. An empty pan was used as a reference.

\section{Rheological Studies}

Rheological analysis of the COW cream and LLC cream was investigated by using an AR 2000ex rheometer (TA Instruments Co. Ltd., USA) with cone-plate geometry of $40 \mathrm{~mm}$ in diameter. Cone angle was set at $2^{\circ}$, and a gap of $0.063 \mathrm{~mm}$ was used. All rheological experiments were carried out at $32^{\circ} \mathrm{C}$.

\section{Flow Curve (Viscometry)}

Pre-shear was done to remove the sample history at loading, then each sample was subjected to a shear rate ranging from 0 to $120 \mathrm{~s}^{-1}$ within $2 \mathrm{~min}$, and the corresponding shear stress $(\mathrm{Pa})$ was measured. The shear rate region was selected on the basis of the sample response to the applied shear stress. Samples were allowed to equilibrate for at least 3 min prior to analysis.

\section{Oscillatory Measurements}

Oscillatory experiments were carried out to study viscoelasticity of the samples. Initially, stress sweep was conducted at a fixed frequency of $1 \mathrm{~Hz}$ in order to determine the linear viscoelastic region (LVR), and the elasticity (storage) modulus $G^{\prime}$ and viscosity (loss) modulus $G^{\prime \prime}$ were measured as a function of the stress amplitude ranging from 0.1 to $100 \mathrm{~Pa}$. Once the LVR was established, frequency sweep was performed over a range of $0.01-100 \mathrm{rad} / \mathrm{s}$, and $3 \mathrm{~Pa}$ was chosen as the controlled stress amplitude, which was within the previously marked linear viscoelastic region for all the samples.

\section{Drug Distribution in Skin, Muscle, and Plasma of Mice Via Topical Administration}

\section{Experimental Protocol}

Seventy-two male mice, in total, were randomly assigned into two groups. For each group, LLC cream or COW cream was applied separately. Before administration, the abdominal

Table I. Factors and Levels in Response Surface Methodology Design

\begin{tabular}{|c|c|c|c|c|c|c|}
\hline \multirow[t]{2}{*}{ Independent variables } & \multirow[t]{2}{*}{ Symbol } & \multicolumn{5}{|c|}{ Levels used, actual (coded) } \\
\hline & & Very low $(-1.732)$ & Low $(-1)$ & Medium (0) & High (1) & Very high (1.732) \\
\hline EA (\%) & $X_{1}$ & 1 & 1.2 & 1.5 & 1.8 & 2 \\
\hline 4-MSK (\%) & $X_{2}$ & 1 & 1.2 & 1.5 & 1.8 & 2 \\
\hline Oil (\%) & $X_{3}$ & 10 & 14.05 & 20.0 & 25.95 & 30 \\
\hline
\end{tabular}


hair of each mouse was removed carefully with an electric clipper and an electric shaver, and then, the skin was washed with normal saline. The mouse with injured skin observed by naked eyes was rejected. A certain amount of cream was applied on the clean skin with an area of $1.5 \times 1.5 \mathrm{~cm}$ which was corresponding to ca. $45 \mathrm{mg} \mathrm{cream} / \mathrm{cm}^{2}$. At predetermined time point of 15, 30, 60, 120, 240, 360, 480, 720 and 1,440 min after administration, four mice from each group were randomly taken out. After blood samples (ca. $0.4 \mathrm{~mL}$ ) were collected via the suborbital veniplex under ether anesthesia, the mice were sacrificed and the residual cream on the skin surface was cleared by cotton swabs impregnated with normal saline immediately. Then, plasma was separated immediately by centrifugation. Subsequently, the skin and muscle at the exact application site were surgically cut off, weighed, and stored at $-70^{\circ} \mathrm{C}$ for further treatment. In the case of sampling at $1,440 \mathrm{~min}$, the cream was removed at $720 \mathrm{~min}$.

\section{Sample Extraction Procedure}

Internal standard solution of $20 \mu \mathrm{L}$ (salicylic acid methanol solution, $258 \mu \mathrm{g} \mathrm{mL}^{-1}$ ) was added to each plasma sample $(100 \mu \mathrm{L})$. The mixture was extracted with $1 \mathrm{~mL}$ of methanol by vortex mixing (MS3, IKA $®$, Germany) for $3 \mathrm{~min}$ and centrifuged $\left(4^{\circ} \mathrm{C}, 16,000 \mathrm{rpm}\right)$ for $10 \mathrm{~min}$. Thereafter, supernatant was transferred into another clean tube and then dried under nitrogen at $40^{\circ} \mathrm{C}$. The residues were redissolved in $100 \mu \mathrm{L}$ methanol by vortex mixing for $3 \mathrm{~min}$ and centrifuged. The final supernatant of $20 \mu \mathrm{L}$ was injected into the HPLC system.

Each weighed skin sample was cut into pieces and collected in a test tube. One milliliter of methanol was added to the tube, and the mixture was sonicated for $20 \mathrm{~min}$ and centrifuged $\left(4^{\circ} \mathrm{C}, 16,000 \mathrm{rpm}\right)$ for $10 \mathrm{~min}$. An equal volume of internal standard solution $\left(20 \mu \mathrm{g} \mathrm{mL}^{-1}\right)$ was mixed with $100 \mu \mathrm{L}$ of the supernatant by vortex mixing for $3 \mathrm{~min}$, and $20 \mu \mathrm{L}$ of the mixture was for HPLC analysis.

The muscle samples were also cut into pieces, extracted with $1 \mathrm{~mL}$ of methanol, sonicated for $20 \mathrm{~min}$, and centrifuged at $4^{\circ} \mathrm{C}, 16,000 \mathrm{rpm}$ for $10 \mathrm{~min}$. The rest of the procedure was the same with that of plasma samples. All tissue and plasma samples were analyzed by a validated HPLC method described as follows.

\section{HPLC Analysis}

The concentrations of EA and 4-MSK in the receptor medium, skin, muscle, and plasma were determined using a HPLC system (Hitachi High-Technologies Corporation, Tokyo, Japan) equipped with an L-2130 pump, an L-2400 ultraviolet absorbance detector, and an L-2200 autosampler. The column used was Spursil ${ }^{\mathrm{TM}}$ C18 column, $5 \mu \mathrm{m}, 150 \times 4.6 \mathrm{~mm}$ (DIKMA Technologies Co., Beijing). The mobile phase consisted of $0.05 \mathrm{~mol} \mathrm{~L}^{-1}$ potassium dihydrogen phosphate in water-methanol $(70: 30 \mathrm{v} / \mathrm{v}$ ) (for biological samples, the ratio of water-methanol was 85:15 $v / v$ ), and it was adjusted to $\mathrm{pH} 7.0$ with triethylamine. The flow rate of the mobile phase was $1 \mathrm{~mL} \mathrm{~min}^{-1}$, and the injection volume was $20 \mu \mathrm{L}$. The sample was detected at $244 \mathrm{~nm}$. The column was maintained at $4^{\circ} \mathrm{C}$. The retention times of EA and 4-MSK were 2.8 and $3.9 \mathrm{~min}$, respectively. All the analytical methods have been validated.

\section{Statistical Data Analysis}

For in vitro skin permeation experiment, the cumulative penetrated amount per unit surface area through the skin within $12 \mathrm{~h}(Q)$ of each drug during each sampling interval was calculated from the measured concentration and volume of the receptor phase. The transdermal penetration curve was obtained when $Q$ was plotted versus time. The steady-state flux $\left(J_{\mathrm{ss}}\right)$ was obtained from the slope of the linear portion of the penetration curve.

The amount of the skin retention $Q_{\mathrm{s}}(\mu \mathrm{g} / \mathrm{g})$ was calculated as follows:

$Q_{\mathrm{s}}=M_{1} / M_{2}$

where $M_{1}$ was the weight of each active ingredient permeated into the skin within $12 \mathrm{~h}$, and $M_{2}$ was the weight of the effective diffusion area of the skin.

All data were calculated and presented as mean \pm SD. Analysis of variance and Student's $t$ test were used for statistical analysis of the data. The level of significance was taken as $p<0.05$.

\section{RESULTS}

\section{Development of the LLC Cream Formulation}

\section{Screening the Type of Oil Via Single-Factor Test}

The result of screening the type of oil was presented in Fig. 2. For EA, the skin retention order was DISM > dimethicone $>$ DIA $>$ Florasun $>$ Floramac, and for 4-MSK, the skin retention order was DISM $>$ dimethicone $>$ Florasun $>$ DIA > Floramac. As DISM provided the best improvement for both EA and 4-MSK, it was chosen as the oil for the final formulation.

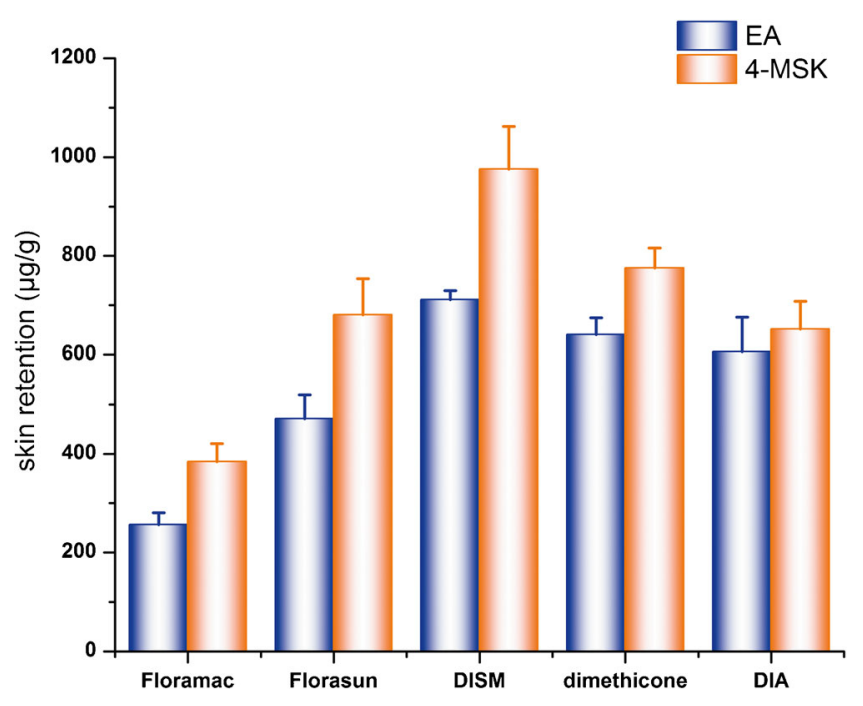

Fig. 2. The skin retention of EA and 4-MSK from various formulations with different kinds of oil ( $n=3$ or 4 ) 


\section{Formulation Optimization by CCD-RSM}

The experimental response result of each formulation was shown in Table II. The experiment data were fitted to multiple nonlinear models using the Design Expert ${ }^{\circledR}$ software. The $p$ values were less than 0.05 , indicating that the model equations were statistically significant. The values of $R^{2}$ for each equation represented the percentage that the selected independent variables contributed to the total variation. The $R$ values for the four response variables were $0.8761,0.8155,0.9444$, and 0.9155 (all higher than 0.8 ), successively, indicating the good correlation of the model equation. The model equations were presented as follows:

$$
\begin{aligned}
& Q_{\mathrm{s} 1}=924.54365-45.47056 \times X_{1} \times X_{2}-6.04301 \times X_{1} \times X_{3}+4.46024 \times X_{2} \times X_{3}\left(p=0.006<0.05, R^{2}=0.8919\right) \\
& Q_{\mathrm{s} 2}=1,206.14610-234.0805 \times X_{1} \times X_{2}+5.64601 \times X_{2} \times X_{3}\left(p=0.0142<0.05, R^{2}=0.8382\right) \\
& Q_{1}=-0.56203+1,031.4897 \times X_{2}-255.24664 \times X_{1} \times X_{2}\left(p=0.0364<0.05, R^{2}=0.7675\right) \\
& Q_{2}=1,957.11583-706.13856 \times X_{1} \times X_{2}-0.035159 \times X_{1} \times X_{2} \times X_{3}+276.85719 \times X_{1} \times X_{2}^{2}\left(p=0.0066<0.05, R^{2}=0.6651\right)
\end{aligned}
$$

The contour plots and three-dimensional (3D) response surfaces shown in Fig. 3 were also fitted to visualize the impact of independent variables on each individual response variable. Taking into consideration that the range of $X_{3}$ was limited for a pleasant skin sensation, $X_{1}$ and $X_{2}$ were selected as independent variables for 3D response surfaces. From Fig. 3 , it can be summarized that the lowest $Q_{1}$ can be obtained in the aera of lower value of $X_{1}$ and $X_{2}$, while the lowest $Q_{2}$ distributed in the aera of the higher value of $X_{1}$ and $X_{2}$. Moreover, the values of $Q_{\mathrm{s} 1}$ and $Q_{\mathrm{s} 2}$ in these ranges were too low. Therefore, according to the principle of maximum $Q_{\mathrm{s} 1}, Q_{\mathrm{s} 2}$ and minimum $Q_{1}, Q_{2}$ with least $X_{1}$ and $X_{2}$, the Design Expert® software was utilized to get a set of optimal value for the formulation, which consisted of $1.20 \%$ of EA, $1.48 \%$ of $4-\mathrm{MSK}$, and $14.05 \%$ of the oil. This process also indicated that interaction effect exists between $X_{1}$ and $X_{2}$. The $X_{3}$ showed relatively less effect on the response variables.
In order to validate the reliability of the optimal values described above, three batches of LLC cream prepared according to the optimized formulation were subjected to the in vitro permeation experiment. As shown in Table III, the deviations between the experimental values and the predicted responses were less than $15 \%$, certifying that the predicted values were in good agreement with the experimental ones. The deviation was calculated as follows:

Deviation $\%=\left(Q_{\text {predicted }}-Q_{\text {experimental }}\right) / Q_{\text {predicted }} \times 100 \%$

where $Q_{\text {predicted }}$ and $Q_{\text {experimental }}$ represented the predicted and experimental values of the cumulative penetrated amount or the skin retention of EA and 4-MSK, respectively.

\begin{tabular}{|c|c|c|c|c|c|c|c|}
\hline Run & $\begin{array}{l}\text { Factor } 1 \\
\text { A: EA }\end{array}$ & $\begin{array}{l}\text { Factor } 2 \\
\text { B: 4-MSK }\end{array}$ & $\begin{array}{l}\text { Factor } 3 \\
\text { C: oil }\end{array}$ & $\begin{array}{l}\text { Response } 1 \\
Q_{1}\end{array}$ & $\begin{array}{l}\text { Response } 2 \\
Q_{2}\end{array}$ & $\begin{array}{l}\text { Response } 3 \\
Q_{\mathrm{s} 1}\end{array}$ & $\begin{array}{l}\text { Response } 4 \\
Q_{\mathrm{s} 2}\end{array}$ \\
\hline 1 & 1.8 & 1.2 & 14.05 & 974.56 & $1,349.2$ & 596.25 & 805.09 \\
\hline 2 & 1.5 & 1.5 & 10.08 & $1,360.5$ & $1,275.5$ & 918.67 & 729.36 \\
\hline 3 & 1.2 & 1.2 & 25.95 & 811.76 & $1,748.9$ & 617.5 & 996.32 \\
\hline 4 & 1.0 & 1.5 & 20.06 & 817.28 & $1,210.8$ & 711.74 & 933.84 \\
\hline 5 & 1.5 & 1.5 & 20.12 & $1,353.2$ & $1,763.6$ & 764.09 & 876.06 \\
\hline 6 & 2.0 & 1.5 & 20.05 & $1,163.4$ & $1,137.3$ & 528.87 & 473.1 \\
\hline 7 & 1.5 & 1.5 & 20.06 & 841.96 & $1,760.7$ & 580.05 & 852.93 \\
\hline 8 & 1.2 & 1.8 & 14.05 & $2,364.4$ & $2,306.4$ & $1,895.9$ & $1,309.6$ \\
\hline 9 & 1.5 & 1.0 & 20.10 & 515.89 & $1,129.2$ & 594.69 & $1,045.3$ \\
\hline 10 & 1.8 & 1.8 & 14.05 & 840.02 & 894.13 & 478.26 & 393.57 \\
\hline 11 & 1.2 & 1.2 & 14.05 & 411.93 & 447.92 & 374.06 & 444.79 \\
\hline 12 & 1.8 & 1.8 & 25.95 & 679.51 & $1,106.7$ & 598.65 & 751.75 \\
\hline 13 & 1.5 & 2 & 20.05 & 721.07 & $1,119.5$ & 564.58 & 629.76 \\
\hline 14 & 1.5 & 1.5 & 20.02 & $1,068.8$ & $1,559.9$ & $1,109.4$ & $1,200.5$ \\
\hline 15 & 1.5 & 1.5 & 30.04 & 802.47 & $1,175.9$ & 732.69 & 699.27 \\
\hline 16 & 1.2 & 1.8 & 25.95 & $1,203.2$ & 1,736 & 824.12 & 849.95 \\
\hline 17 & 1.8 & 1.2 & 25.95 & 600.42 & 898.62 & $1,281.3$ & $1,439.1$ \\
\hline
\end{tabular}
Therefore, the final formulation contained $1.20 \%$ of EA, $1.48 \%$ of $4-\mathrm{MSK}$, and $14.05 \%$ of oil.

Table II. Scheme of Response Surface Methodology with the Results of Responses on Three Independent Factors

EA 3-O-ethyl-ascorbic acid, 4-MSK potassium 4-methoxysalicylate, $Q_{1}$ cumulative penetrated amount of EA ( $\left.\mu \mathrm{g} / \mathrm{cm}^{2}\right), Q_{2}$ cumulative penetrated amounts of 4-MSK $\left(\mu \mathrm{g} / \mathrm{cm}^{2}\right), Q_{s 1}$ skin retention of EA $(\mu \mathrm{g} / \mathrm{g}), Q_{s 2}$ skin retention of 4-MSK $(\mu \mathrm{g} / \mathrm{g})$ 
a

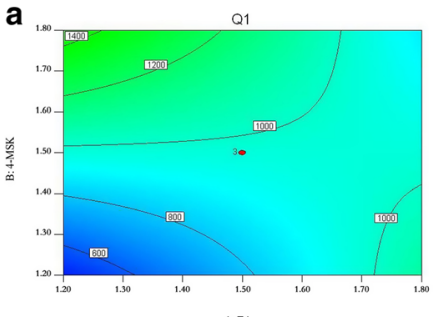

$\bar{\sigma}$

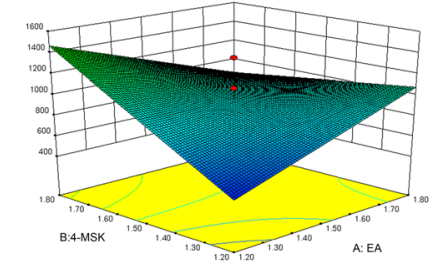

b
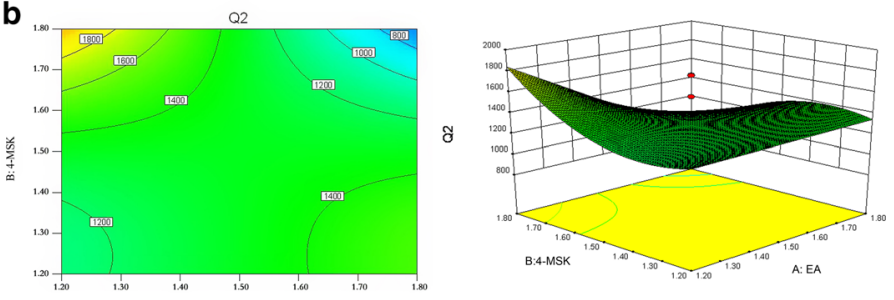

C

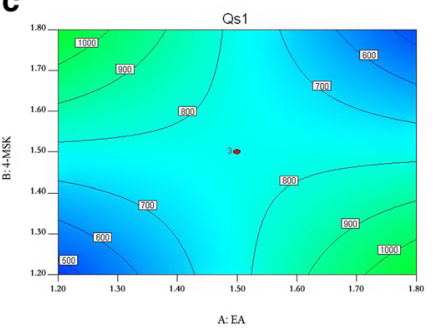

$\overline{5}$

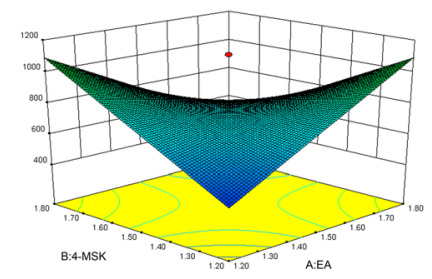

d

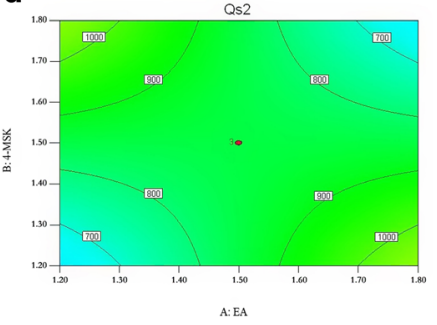

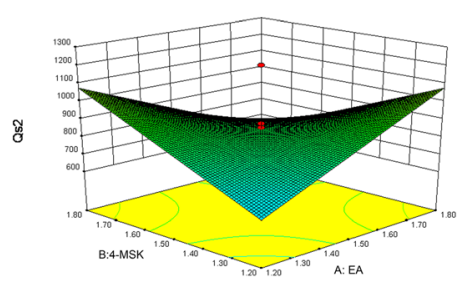

Fig. 3. Contour plots and three-dimensional (3D) response surface model showing the influence of independent variables on the response variables: a cumulative penetration amounts of EA $\left(Q_{1}\right)$; b the cumulative penetration amounts of 4$\operatorname{MSK}\left(Q_{2}\right) ; \mathbf{c}$ the skin retention of EA $\left(Q_{\mathrm{s} 1}\right) ; \mathbf{d}$ the skin retention of 4-MSK $\left(Q_{\mathrm{s} 2}\right)$

\section{Characterization of LLC Cream}

$P L M$

Polarized light microscopy can be used to demonstrate the existence of LLC phase. As shown in Fig. 4, the samples of LLC cream presented Maltese crosses, while the COW cream displayed a dark field under polarized light.

\section{$D S C$}

The thermodynamic behavior of the two types of cream was studied by DSC. The DSC curve (shown in Fig. 5) of the

Table III. The Result of Response Surface Methodology Validation Experiment

\begin{tabular}{lcllcc}
\hline Responses & Predicted values & Opt $1^{a}$ & Opt $2^{a}$ & Opt 3 & Deviation (\%) \\
\hline$Q_{\mathrm{s} 1}$ & 833.78 & 807.28 & 784.62 & 899.49 & 0.40 \\
$Q_{\mathrm{s} 2}$ & 904.39 & 936.98 & $1,041.25$ & 926.00 & 7.04 \\
$Q_{1}$ & $1,076.25$ & 955.11 & 925.97 & 911.09 & 13.52 \\
$Q_{2}$ & $1,428.32$ & $1,615.14$ & $1,647.59$ & $1,516.8$ & 11.54 \\
\hline
\end{tabular}

${ }^{a}$ Opt 1 , opt 2, and opt 3 represent the experimental values of the cumulative penetrated amount or the skin retention of EA or 4-MSK from the three batches of LLC cream prepared according to the optimized formulation 
a

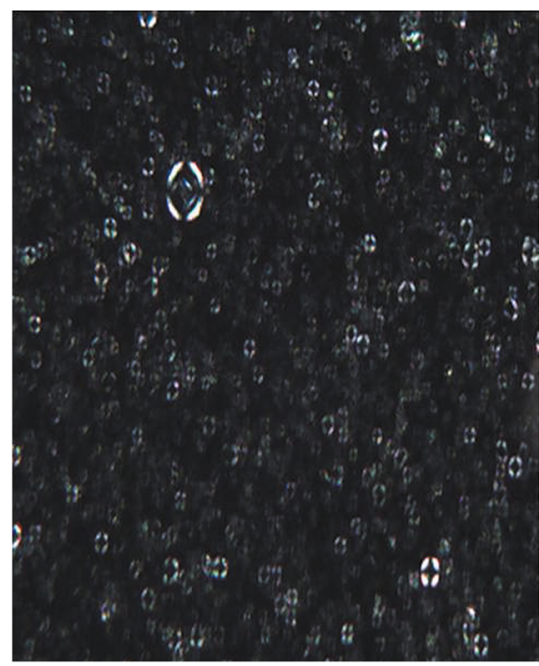

b

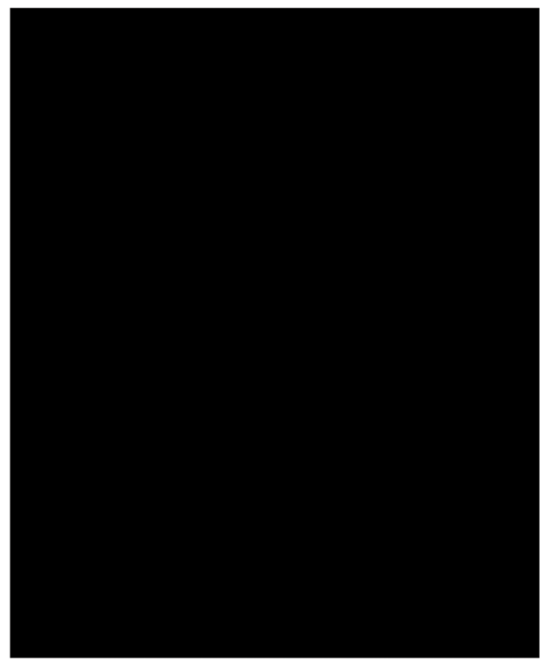

Fig. 4. Polarized light microscopy photomicrographs of the LLC cream (a) and the COW cream (b)

LLC cream exhibited two endothermic peaks at $-0.42^{\circ} \mathrm{C}$ and $54^{\circ} \mathrm{C}$, while that of the COW cream presented only one endothermic peak at $-5.92^{\circ} \mathrm{C}$.

\section{Rheological Studies}

The flow curves of shear viscosity versus shear rate for the COW cream and the LLC cream were shown in Fig. 6. The viscosity of the two kinds of cream was almost the same.

As shown in Fig. 7, the bigger hysteresis loop of the LLC cream revealed its stronger thixotropy than that of the $\mathrm{COW}$ cream.

Frequency-dependent rheological experiments are widely used to characterize the viscoelasticity of the LLC system. In the tests, $G^{\prime}$ and $G^{\prime \prime}$ remained constant up to a critical stress of $10 \mathrm{~Pa}$ in all the cases. Consequently, $3 \mathrm{~Pa}$ was chosen as the stress amplitude for the subsequent dynamic experiments to guarantee a liner viscoelastic response for all the samples.

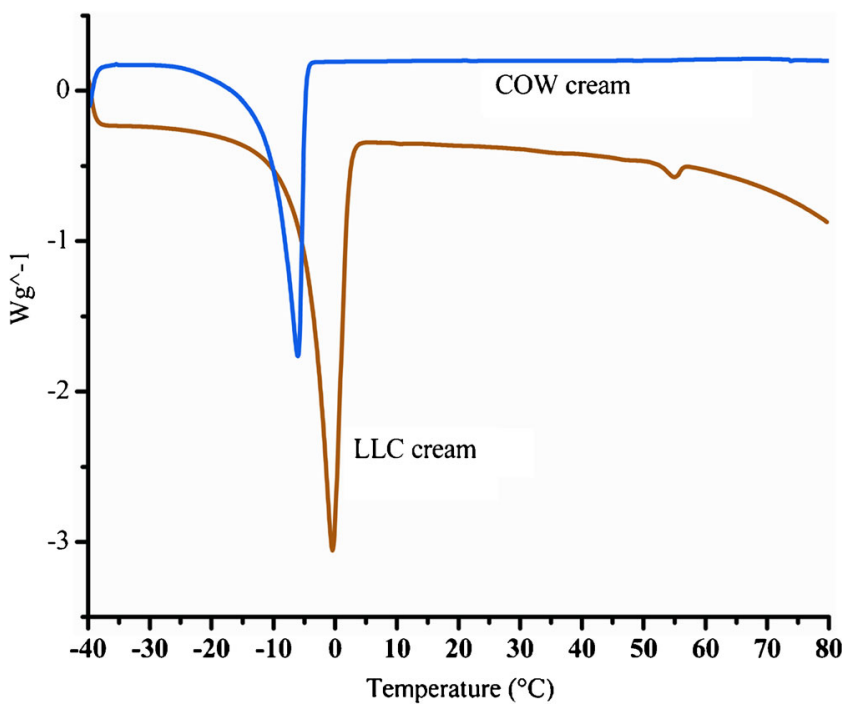

Fig. 5. DSC curve of the LLC cream and COW cream at a heating rate of $5^{\circ} \mathrm{C} \mathrm{min}-1$
Figure 8 showed the typical results of $G^{\prime}$ and $G^{\prime \prime}$ as a function of the frequency in the LVR. The LLC cream turned out to be more elastic than viscous $\left(G^{\prime}>G^{\prime \prime}\right)$ in the whole frequency range, and $G^{\prime}$ was about one order of magnitude higher than $G^{\prime \prime}$. Moreover, $G^{\prime}$ had a dependence on the applied frequency and increased slightly while $G^{\prime \prime}$ showed a minimum. All the abovementioned characteristics confirmed the existence of lamellar liquid crystalline structure of the LLC cream samples.

\section{LLC Cream Increased the Skin Retention of Drugs Both In Vitro and In Vivo}

To assess whether the LLC carrier could facilitate the skin retention of drugs, the permeability of EA and 4-MSK from the LLC cream and the COW cream was compared through in vitro skin permeability experiment with the same amount of the active ingredients. As shown in Fig. 9, the LLC

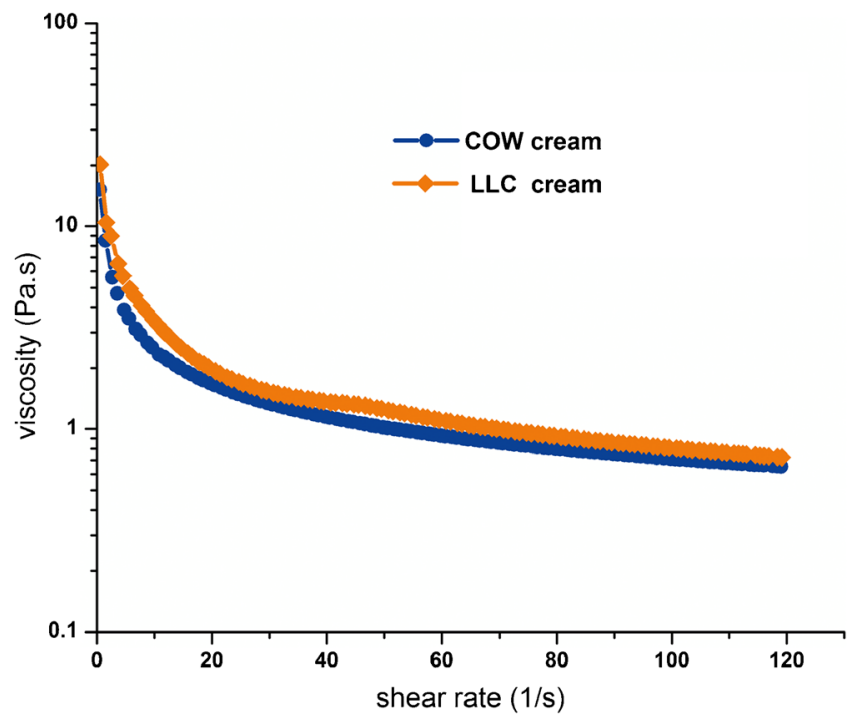

Fig. 6. The flow curve of shear viscosity versus shear rate for the LLC cream and the COW cream 


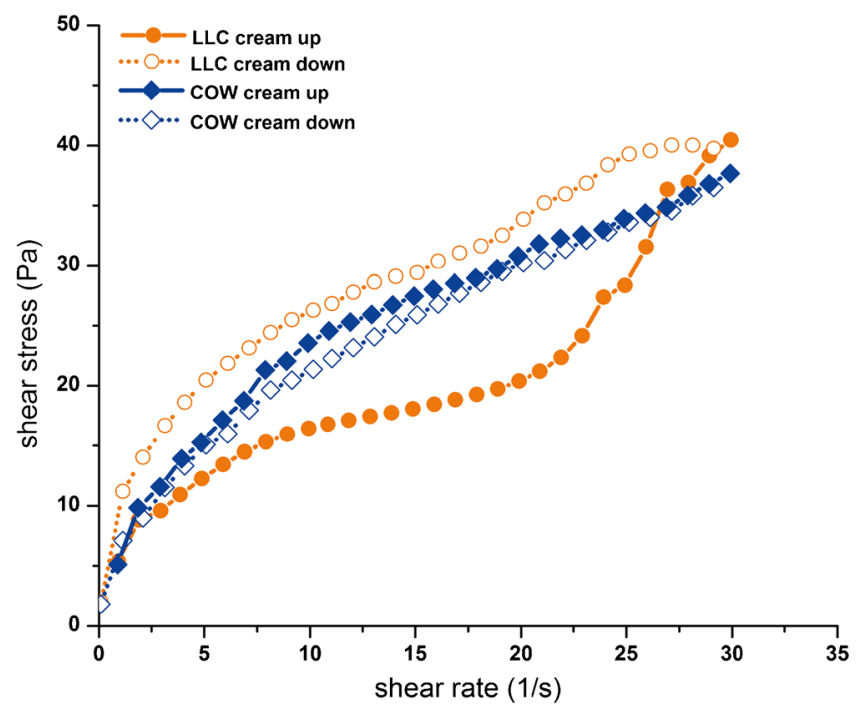

Fig. 7. The thixotropic characteristics of the LLC cream and the COW cream

carrier significantly increased the skin retention of EA and 4MSK by 2.62- and 2.22-fold, respectively. Additionally, Fig. 10 suggested that both creams provided sustained stable permeation through excised rabbit skin within $12 \mathrm{~h}$.

To further confirm the effectiveness of the LLC cream, a drug distribution investigation was carried out in KM mice. Figure 11 showed the drug concentration profiles in skin, muscle, and plasma after topical administration of the LLC cream or the COW cream. It was found that the LLC cream delivered much higher amount of drugs into the skin over a range of 0.25 to $8 \mathrm{~h}$ as compared to the COW cream, demonstrating that the cutaneous absorption of both drugs can be improved significantly by the LLC carrier.

The LLC cream delivered significantly higher levels of drugs into the skin than into the muscle $(p<0.05)$, indicating that high percentage of the drugs could retain in the target action site, i.e., skin, which was essential for an effective skinwhitening agent. The content of EA in the skin maintained at

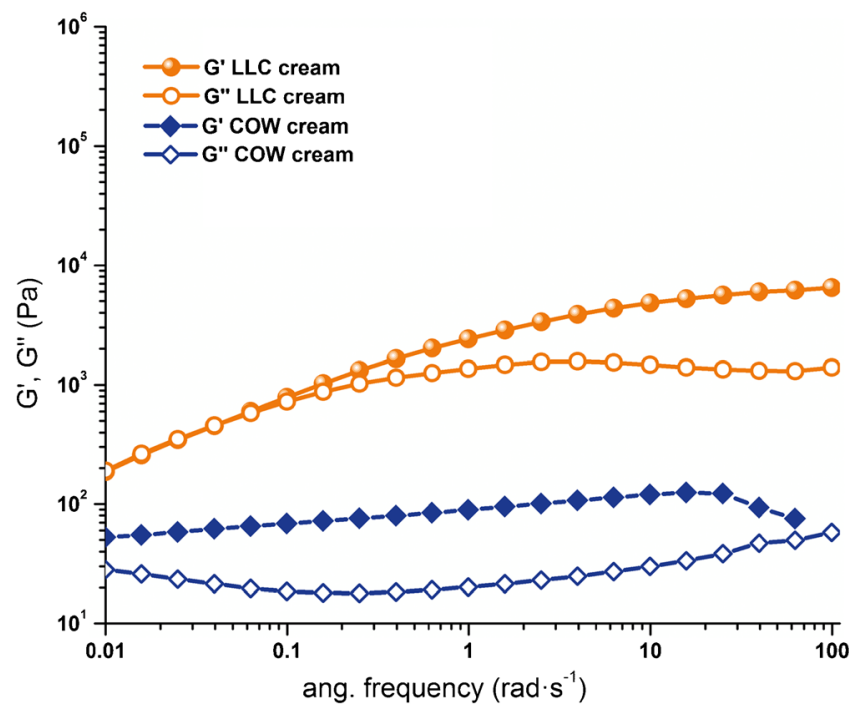

Fig. 8. Result of frequency sweep experiment at a constant stress of $3 \mathrm{~Pa}$ for the two kinds of cream a high level within $12 \mathrm{~h}$. And only $8.42 \pm 4.15 \mu \mathrm{g} / \mathrm{g}$ of EA was detected in the skin at the time of $24 \mathrm{~h}$ after the removal of the cream at $12 \mathrm{~h}$. The trend of content change of 4-MSK in skin over time was similar as EA, except for the slower elimination rate from skin after the removal of cream.

The muscle concentration curves of EA and 4-MSK of the LLC cream were flatter than those of the COW cream, and for 4-MSK, the peak value of the COW cream was even higher than that of the LLC cream. Both of EA and 4-MSK were at very low levels in the plasma from the two kinds of cream. Therefore, both of the cream showed desirable low systemic exposure.

\section{DISCUSSION}

\section{Characterization of LLC Cream PLM}

The anisotropic lyotropic liquid crystals can be distinguished by the optical properties of birefringence due to their molecular organization, and the lamellar liquid crystals characterize by Maltese crosses texture under polarized light $(12,13)$. However, isotropic materials just show a dark region under polarized light, for they have the same optical properties in all directions.

$D S C$

The peak at $-0.42^{\circ} \mathrm{C}$ was due to the "melting" of bulklike water located between the bilayers of the lamellar liquid crystals (14), and peak at $54^{\circ} \mathrm{C}$ represented the gel-liquid crystal transition, possibly a result of the "melting" of the hydrocarbon tails of the surfactant in the bilayers from an ordered structure to a more disordered state. Similar results have also been reported previously, in which this phenomenon was explained as thermal transitions in surfactant-based lyotropic liquid crystals (15). The gel-liquid crystal transition as well as phase transition between different liquid crystalline phases consumed low amount of energy; therefore, only a small endothermic peak occurred at $54^{\circ} \mathrm{C}$ (12). The peak at $-5.92^{\circ} \mathrm{C}$ of the COW cream must be the result of "melting" of the interfacial water, which "melts" easier than the bulk-like water (14).

\section{Rheological Studies}

Proper rheological characteristics including the viscosity, thixotropy, and viscoelasticity are critical for the utilization of a topical cream. In addition, the viscosity of the matrix can inversely affect the release of drugs from the base, thus influencing the skin penetration behavior of drugs (16). Therefore, the rheological characteristics of the two kinds of cream were investigated here.

As compared with COW cream, it seemed that the LLC carrier did not alter the viscosity of the cream, and it was assumed that the tiny viscosity difference between the two kinds of cream would not affect their permeation behavior. The flow profile of both creams exhibited shear-thinning behavior with increasing shear rate, suggesting their internal network structure was gradually broken and turned into a more organized fluid structure 

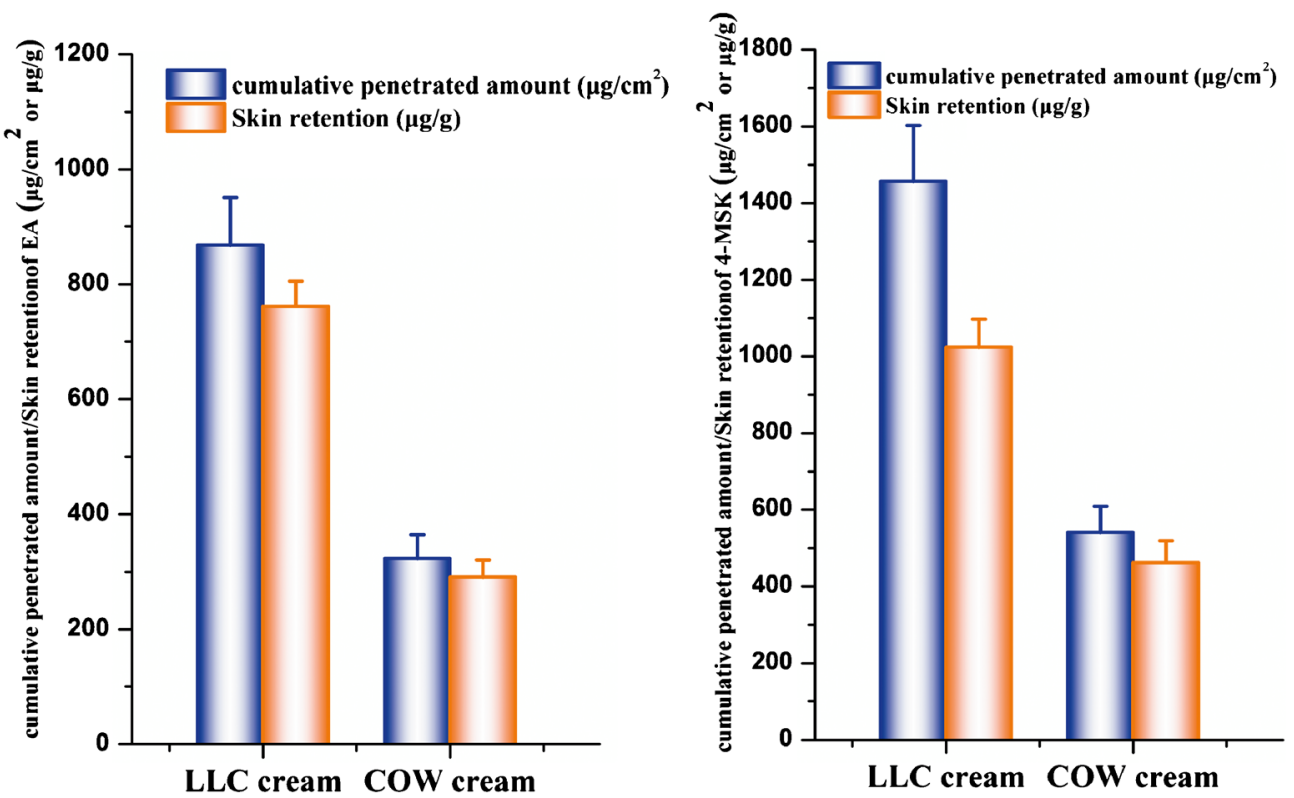

Fig. 9. Comparison of cumulative penetrated amount and skin retention of EA and 4-MSK between the LLC cream and the COW cream via in vitro permeation experiment ( $n=3$ or 4$)$

with the shear rate variations. And after applying a tension, the curve became smooth, indicating the creams were easy to spread uniformly on the skin surface.

Thixotropic characteristics can be observed through continuous ramp up and down test, in which the shear rate sweeps from zero to a maximum value and subsequently decreases back to zero in the same way. A time-dependent change in viscosity occurred, with the above shear-thinning phenomenon under pressure, and returned to the original state over time when the pressure was removed. Shear-thinning under pressure facilitates the product application and skin compliance, while structure recovery after administration allows an efficient bioadhesion; hence, the LLC cream with stronger thixotropy is preferable (9).
In oscillatory experiments, $G^{\prime}$ of COW cream was also higher than $G^{\prime \prime}$ but the difference was smaller than the LLC cream, indicating both of the cream behaved more like elastic solid but the LLC cream had more stable microstructure. It seemed that the presence of LLC carrier resulted in enhanced elasticity and increased solid-like response. These results were consistent with those reported by Makai et al. (17) and Yariv et al. (18).

The rheological difference between the LLC cream and the COW cream revealed the superiority of the LLC cream as a preparation, for LLC carrier offered stronger thixotropy, good stability, and high skin affinity, which can be attributed to the similarity with the skin microstructure.
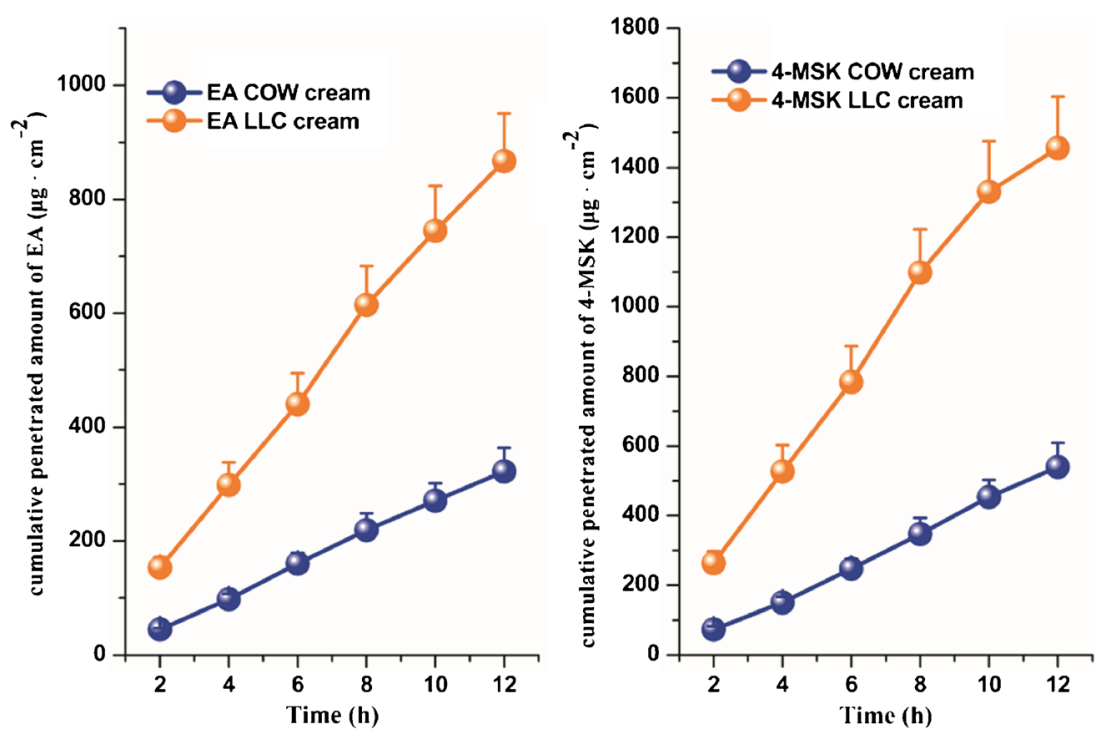

Fig. 10. Permeation profiles of EA and 4-MSK from the LLC cream and COW cream through in vitro permeation experiment ( $n=3$ or 4 ) 
a
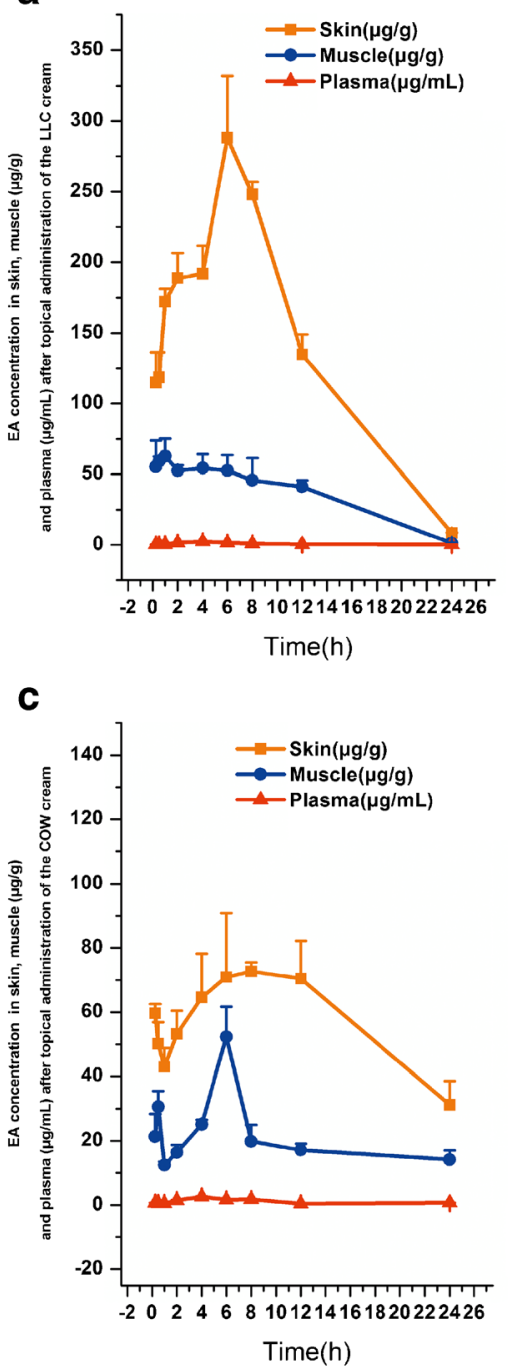

b

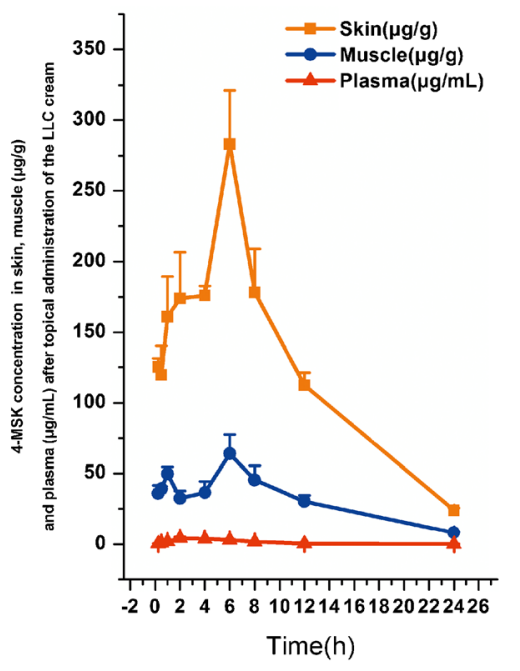

d

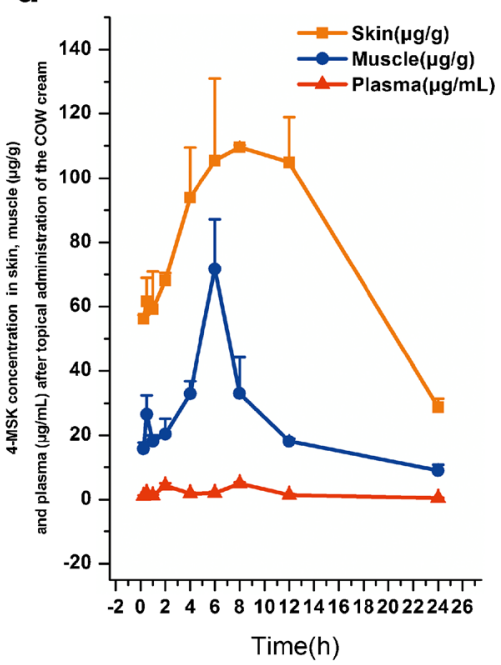

Fig. 11. The concentration of the two drugs in skin, muscle, and plasma of KM mice after topical administration of the two kinds of cream. a EA of the LLC cream; b 4MSK of the LLC cream; $\mathbf{c}$ EA of the COW cream; $\mathbf{d}$ 4-MSK of the COW cream $(n=3$ or 4)

\section{LLC Cream Increased the Skin Retention of Drugs Both In Vitro and In Vivo}

The results of in vitro skin permeability experiment revealed LLC cream increased the skin retention of drugs in vitro. This finding was consistent with the previous published work related to an anti-wrinkle drug of Iwai et al. (19). To our knowledge, drug distribution in the skin, muscle, and plasma of the LLC cream via topical administration has not been done previously.

Liquid crystals are matter in an intermediate state the fluid liquid and the anisotropic crystal. The lamellar liquid crystal is parallel planar bilayer of amphiphilic molecules separated by layers of water with each bilayer similar to the arrangement of lipids in cell membranes. The above results could be attributed to the superior characteristics of the liquid crystalline carrier system. Firstly, since large amount of water was tightly incorporated between the hydrophilic layers of the LLC system, the evaporation of water from the system is much slower than that from the COW cream, thus leading to a long-lasting moisturizing effect on the skin (20). As is known, the moisture can elicit skin hydration associated with high drug permeability and a pleasant skin sensation. Moreover, the LLC carrier exhibited better skin permeability probably due to interactions between the microstructure of liquid crystalline emulsifier and the intercellular lipids in the SC, which yield a more fluid and permeable lipid packing $(21,22)$. Furthermore, the very low oil/water interfacial surface tension induced by the liquid crystalline emulsifier may also facilitate the progressive permeation of the drugs into the skin (23). Most importantly, the lamellar system possesses structure similar to the lipid bilayer of the cell membrane, hence showing a high affinity to the skin. Consistent with previous other works, all the above advantages make the lamellar liquid crystal a great carrier system. 


\section{CONCLUSION}

In the present study, the LLC cream exhibited improved skin retention in vitro and in vivo as compared to the $\mathrm{COW}$ cream. The LLC cream delivered more drugs into the skin than into the muscle, with low systematic exposure. Rheological experiments revealed that the LLC cream possessed more stable structure, and its stronger thixotropy indicated better skin-compliant and skin application characteristics. Hence, it can be concluded that the LLC cream with the optimized formulation is promising for depigmentation or skin-whitening, and the LLC carrier can serve as a candidate to increase the skin retention of drugs for topical administration.

\section{ACKNOWLEDGMENTS}

This work was supported by the Students' Innovation and Entrepreneurship Training Program of China.

Conflict of Interest The authors declare that they have no competing interests. The authors alone are responsible for the content and writing of this article.

\section{REFERENCES}

1. Desmedt B, Van Hoeck E, Rogiers V, Courselle P, De Beer J, De Paepe K, et al. Characterization of suspected illegal skin whitening cosmetics. J Pharm Biomed. 2014;90:85-91.

2. Arbab A, Eltahir M. Review on skin whitening agents. Khartoum Pharm J. 2010;13(1):5-9.

3. Hatao M, Maeda K. Method of preventing darkening of skin or inhibiting melanization of melanin monomer and polymerization inhibitor of biological dihydroxyindole compound. Google Patents; 2005.

4. Isoda M, Ueda S, Imayama S, Tsukahara K. New formulation of chemical peeling agent: histological evaluation in sun-damaged skin model in hairless mice. J Dermatol Sci. 2001;27:60-7.

5. Tojo K, Chiang C, Chien Y. Drug permeation across the skin: effect of penetrant hydrophilicity. J Pharm Sci. 1987;76(2):123-6.

6. Brinon L, Geiger S, Alard V, Doucet J, Tranchant J-F, Couarraze G. Percutaneous absorption of sunscreens from liquid crystalline phases. J Control Release. 1999;60(1):67-76.

7. Nesseem DI. Formulation and evaluation of itraconazole via liquid crystal for topical delivery system. J Pharm Biomed. 2001;26(3):387-99.
8. Hosmer JM, Shin SH, Nornoo A, Zheng H, Lopes LB. Influence of internal structure and composition of liquid crystalline phases on topical delivery of paclitaxel. J Pharm Sci. 2011;100(4):144455 .

9. Chorilli M, Prestes P, Rigon R, Leonardi G, Chiavacci L, Sarmento $\mathrm{V}$, et al. Structural characterization and in vivo evaluation of retinyl palmitate in non-ionic lamellar liquid crystalline system. Colloids Surf B Biointerfaces. 2011;85(2):182-8.

10. Benton WJ, Miller CA, Fort Jr T. Spontaneous emulsification in oil-water-surfactant systems. J Dispers Sci Technol. 1982;3(1):144.

11. Xi H, Cun D, Xiang R, Guan Y, Zhang Y, Li Y, et al. Intraarticular drug delivery from an optimized topical patch containing teriflunomide and lornoxicam for rheumatoid arthritis treatment: does the topical patch really enhance a local treatment? J Control Release. 2013;169(1):73-81.

12. Müller-Goymann C. Physicochemical characterization of colloidal drug delivery systems such as reverse micelles, vesicles, liquid crystals and nanoparticles for topical administration. Eur J Pharm Biopharm. 2004;58(2):343-56.

13. Muniandy S, Kan C, Lim S, Radiman S. Fractal analysis of lyotropic lamellar liquid crystal textures. Phys A Stat Mech Appl. 2003:323:107-23.

14. Casillas N, Puig JE, Olayo R, Hart TJ, Franses EI. State of water and surfactant in lyotropic liquid crystals. Langmuir. 1989;5(2):384-9.

15. Schulz P, Puig J, Barreiro G, Torres L. Thermal transitions in surfactant-based lyotropic liquid crystals. Thermochim Acta. 1994;231:239-56.

16. Li C, Liu C, Liu J, Fang L. Correlation between rheological properties, in vitro release, and percutaneous permeation of tetrahydropalmatine. AAPS PharmSciTech. 2011;12(3):100210.

17. Makai M, Csányi E, Németh Z, Palinkas J, Erôs I. Structure and drug release of lamellar liquid crystals containing glycerol. Int J Pharm. 2003;256(1):95-107.

18. Yariv D, Efrat R, Libster D, Aserin A, Garti N. In vitro permeation of diclofenac salts from lyotropic liquid crystalline systems. Colloids Surf B Biointerfaces. 2010;78(2):18592.

19. Iwai H, Fukasawa J, Suzuki T. A liquid crystal application in skin care cosmetics. Int J Cosmet Sci. 1998;20(2):87-102.

20. Rawlings A, Harding C. Moisturization and skin barrier function. Dermatol Ther. 2004;17(s1):43-8.

21. Hosmer JM, Steiner AA, Lopes LB. Lamellar liquid crystalline phases for cutaneous delivery of paclitaxel: impact of the monoglyceride. Pharm Res. 2013;30(3):694-706.

22. Otto A, Du Plessis J, Wiechers J. Formulation effects of topical emulsions on transdermal and dermal delivery. Int J Cosmet Sci. 2009;31(1):1-19.

23. Suzuki T, Takei H, Yamazaki S. Formation of fine three-phase emulsions by the liquid crystal emulsification method with arginine $\beta$-branched monoalkyl phosphate. J Colloid Interface Sci. 1989;129(2):491-500. 